\title{
Improving Optical-Wireless CDMA System Performance in Indus- trial Environment with Timing Jitters
}

\author{
Yen-Ting Lin ${ }^{\mathrm{a}}$, Guu-Chang Yang ${ }^{\mathrm{a},{ }^{*}}$, Cheng-Yuan Chang ${ }^{\mathrm{b}}$, Min-Kuan Chang ${ }^{\mathrm{a}}$, Wing C. Kwong ${ }^{\mathrm{c}}$ \\ ${ }^{\mathrm{a}}$ Department of Electrical Engineering, National Chung Hsing University, Taichung 402, Taiwan, R.O.C. \\ ${ }^{\mathrm{b}}$ Department of Electrical Engineering, National United University, Miaoli 360, Taiwan, R.O.C. \\ ${ }^{\mathrm{c}}$ Department of Engineering, Hofstra University, Hempstead, NY 11549, U.S.A. \\ *Corresponding Author: gcyang@dragon.nchu.edu.tw
}

\begin{abstract}
In this paper, a novel analytical model is proposed and formulated to quantify the timing jitters, introduced by environmental changes, in optical-wireless code-division multiple access systems. The model divides every chip in an optical codeword into multiple equal intervals, and each pulse in the codeword can be randomly shifted to one of these sub-chip positions in order to account for the effect of the timing jitters. Our study shows that the new model can make a good use of the time skew of pulses in optical codewords and unconventionally improve O-CDMA performance under a certain condition.
\end{abstract}

Keywords: optical code-division multiple access, optical wireless, temperature fluctuations, timing jitters.

\section{Introduction}

Direct-detection (or so-called incoherent) optical code division multiple access (O-CDMA) has been studied for applications in fiber-optic and optical-wireless multipleaccess systems and networks because of its desirable features, such as flexible bandwidth utilization, asynchronous access without the need of precise coordination, efficiency in bursty traffic, and dynamic optical-channel sharing without complex scheduling ${ }^{(1-4)}$. While CDMA has already been used in wireless communications, its usefulness in industrial/manufacturing plants that see strong electromagnetic interference (EMI) is moot. As optical technology is immune to EMI, the use of CDMA in optical-wireless computer/communications/control networks in such strong-EMI environment becomes attractive. Also, optical-wireless CDMA can also provide mobility and ease of set-up/tear-

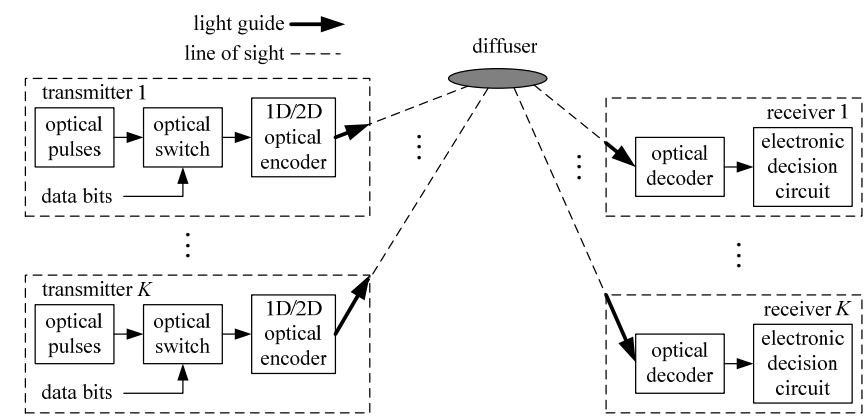

Fig. 1 Example of an optical-wireless CDMA system using free space as the multi-access optical channel, suitable for manufacturing plants that have strong EMI and prefer node mobility.

$\operatorname{down}^{(5-8)}$.

Figure 1 shows an example of an optical-wireless CDMA system model, in which optical codeword of each user (or node) is transmitted via free space for mobility and EMI immunity. Assume that every user sends its data bits in on off keying (OOK) modulation format. In the transmitter of a node, a gated optical pulse, representing the transmission of a data bit of 1 , is first encoded into the address codeword of the receiver of the intended node. The structure of the 1-D/2-D optical encoders depends on the 1-D or 2-D coding scheme in use. The codeword is then transmitted onto the diffuser(s) on the ceiling and, in turn, distributed to all nodes. In each receiver, the optical decoders serve as inverted filters of the optical encoders. Each decoder matches the time positions (and wavelengths, if 2-D wavelength-time codes are used) of the pulses of arriving codewords with its address signature. A hard-limiter can be placed at the front end of each optical decoder to reduce MAI-localization and near-far problems ${ }^{(9,10)}$.

Two important issues in optical-wireless CDMA are on the codeword arrival-time tracking and integrity of the op- 
tical pulses (i.e., precisely sitting in their associated chips or time slots) within each codeword. It is commonly assumed that the arrival time of the codewords from a node is fixed after the timing of the node has been established in the system. Nevertheless, physical or environmental changes, such as temperature fluctuations, may cause timing jitters and, in turn, generate a slight mismatch in the codeword's arrival time at the intended receiver and also create time skew on the optical pulses within the codeword. For example, the effect of environmental temperature fluctuations to the performance of a long-haul fiber-optic CDMA system was studied by Osadola, et al. in ${ }^{(11)}$. They included fiber thermal coefficient in the analysis and modeled the effect of temperature variations as time skew on the optical pulses in codewords. The distortion of autocorrelation peaks and, in turn, worsening of system performance were formulated as a function of the amount of time skew introduced by temperature fluctuations and distance traveled. Even though their study was not directly applicable, it raised an important fact that any time skew on the optical pulses within transmitted codewords would cause performance degradation in optical-wireless CDMA systems as well.

However, their analytical model did not take into account that the cross-correlation properties of the optical codewords in use could also be changed by time skew, in addition to the autocorrelation peaks. Our study in Section 2 shows that time skew indeed worsens the cross-correlation values of the optical codes in use. In Section 3, a novel analytical model, which can be used to complement the model in ${ }^{(11)}$, is formulated. Our results imply that time skew of optical pulses, caused by environmental changes or timing jitters, can constructively be exploited to improve system performance if there exists a feedback mechanism in the receiver to reconstruct the original autocorrelation peak. This new finding is unconventional in the sense that an O-CDMA system can make good use of this deleterious effect to improve performance, rather than harming it, opposite to the finding in ${ }^{(11)}$. Finally, the new model is validated by numerical examples and computer simulation in Section 4.

\section{Quantifying Time Skew and Its Effect to Cross Correlations}

In incoherent O-CDMA with OOK modulation, each user conveys the address codeword of its intended receiver whenever a data bit of 1 is transmitted, but nothing is conveyed for bits of 0 . Assume the use of a family of $(L \times N, w$,

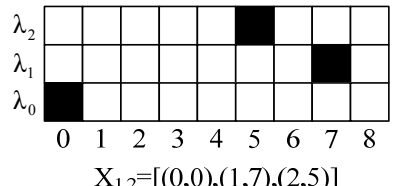

$\mathrm{X}_{1,2}=[(0,0),(1,7),(2,5)]$

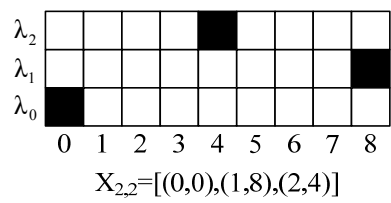

(a)

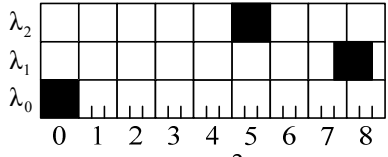

$\left[(0,0),\left(1,7+\frac{2}{3}\right),(2,5)\right]$

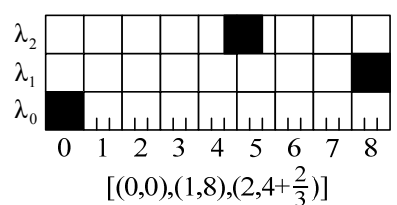

(b)
Fig. 2 Example of how the random time-shifts affect the cross-correlation function between the $(3 \times 9,3,1) 2$-D CHPC codewords $X_{1,2}=[(0,0),(1,7),(2,5)]$ and $X_{2,2}=[(0,0),(1,8),(2$, $4)]$ with $s=3$ : (a) the original codewords without any random pulse time-shifts, resulting in the amount of pulse overlap of at most 1 in their cross-correlation function; (b) the shifted copies of both codewords increase the amount of pulse overlap up to $2 \frac{1}{3}$.

$\lambda_{\text {c }}$ 2-D optical codes, which consists of $L \times N$ binary codewords with $L$ wavelengths, $N$ time slots (or code length), $w$ pulses (or code weight), and the maximum cross-correlation value of $\lambda_{\mathrm{c}}{ }^{(2,4)}$. In general, every $2-\mathrm{D}$ codeword, say codeword $i$, in the code set can be represented its $w$ pulses in form of $w$ ordered pairs such that $C_{i}=\left[\left(\lambda_{0}, t_{0}\right),\left(\lambda_{1}, t_{1}\right), \ldots\right.$, $\left.\left(\lambda_{w-1}, t_{w-1}\right)\right]$, where each ordered pair denotes that the pulse of wavelength $\lambda_{j}$ is located in time-slot (or chip) position $t_{j}$ $\in\{0,1, \ldots, N-1\}$ for all $j \in\{0,1, \ldots, w-1\}$. For the case of 1 -D optical codes, $L=1$ and all $w$ pulses in every codeword in the code set use one identical wavelength such that $\lambda_{0}=$ $\lambda_{1}=\cdots=\lambda_{w-1}$.

Due to temperature fluctuations, the time skew of optical pulses can here be quantified as random time shift. In our model, the chips (or time slots) of the optical pulses in every codeword is subdivided into $s$ sub-chips of equal width, where $s>1$ is an integer. Each of these $w$ pulses can be randomly shifted to start at any one of these $s$ sub-chips from its original chip. Let the width of every chip be equal to 1 . Then, the width of every sub-chip is $1 / s$. Also let the time delay of the $j$ th pulse (for 1-D codes) or the pulse of the $j$ th wavelength (for 2-D codes) created by an independent random sub-chip shift be denoted as $\tau_{j} \in\{0,1 / s, 2 / s, \ldots$, $(s-1) / s\}$ for all $j \in[0, w-1]$. So, the ordered pairs of the "shifted" copy of $C_{i}$ become $\left[\left(\lambda_{0}, t_{0}+\tau_{0}\right),\left(\lambda_{1}, t_{1}+\tau_{1}\right), \ldots,\left(\lambda_{w-1}\right.\right.$, $\left.\left.t_{w-1}+\tau_{w-1}\right)\right]$.

Due to the random shifts of the pulses, the cross-correlation property of the optical codes in use is changed and this, in turn, affects the code performance, both now becoming a function of $s$. 
To illustrate the model and how the cross-correlation function gets worsened, the use of the $(L \times N, w, 1) 2$-D carrier hopping prime codes (CHPCs) is here assumed because the codes have many desirable features, for example, its algebraic construction algorithm can be easily modified to generate various families of optical codes with different code properties $^{(12,13)}$. Also, each CHPC codeword consists of at most one pulse per row and per column, and each pulse is assigned with a distinct wavelength, thus giving $\lambda_{\mathrm{c}}=1$.

Using $k=2, w=p_{1}=p_{2}=3$ as an example, the $(3 \times 9,3,1)$ 2-D CHPC has 9 codewords, denoted $X_{i 1, i 2}$ for $i_{1} \in[0,2]$ and $i_{2} \in[0,2]$, represented by the ordered pairs [ $(0,0),(1$, $\left.i_{1}+i_{2} p_{1}\right),\left(2,2 \odot p_{1} i_{1}+\left(2 \odot p_{2} i_{2}\right) p_{1}\right]$. Although these original CHPC codewords have the cross-correlation functions of at most one (i.e., $\left.\lambda_{c}=1\right)^{(2, \text { pp. 84-86) }}$, the random time shifts in the pulses of the codewords will break the pseudo orthogonality of the codes. Figure 2 illustrates how the random timeshifts worsen the cross-correlation function between the $(3 \times$ 9, 3, 1) 2-D CHPC codewords $X_{1,2}=[(0,0),(1,7),(2,5)]$ and $X_{2,2}=[(0,0),(1,8),(2,4)]$ with $s=3$, where the black squares represent the chip locations and carrier wavelengths of the pulses of the codeword. (The vertical axis denotes wavelengths and the horizontal axis denotes time-slots or chips.) While Figure 2(a) shows the original codewords $X_{1,2}$ and $X_{2,2}$, Figure 2(b) shows a shifted copy of $X_{1,2}$ with the time delays $\tau_{0}=0, \tau_{1}=2 / 3$, and $\tau_{2}=0$, resulting in the ordered pairs $[(0,0),(1,7+2 / 3),(2,5)]$ in the shifted codeword, and a shifted copy of $X_{2,2}$ with the time delays $\tau_{0}=0, \tau_{1}=0$, and $\tau_{2}=$ $2 / 3$, resulting in the ordered pairs $[(0,0),(1,8),(2,4+2 / 3)]$ in the shifted codeword.

In the above example, the cross-correlation process between the shifted copies of two codewords can created as large as $2 \frac{1}{3}$ pulse-overlaps (or so-called hits), even though the original cross-correlation function of the codes was at most 1 (i.e., $\lambda_{\mathrm{c}}=1$ ). In general, for a given $s>1$, it is found that the maximum cross-correlation value of the $\left(w \times p_{1} p_{2} \cdots\right.$ $\left.p_{k}, w, 1\right)$ CHPCs is upper bounded by

$$
\lambda_{c} \leq 3-\frac{2}{s}
$$

where $w \geq 3$. This upper bound can also be applied to any 1 $\mathrm{D}$ and 2-D optical codes that each of the codewords uses at most one pulse per wavelength and per chip, and no wavelengths are used more than once within each codeword.

\section{Performance Analysis}

With $s>1$, the optical pulses in each codeword are assumed to be independently and randomly shifted by some integral multiples of $1 / s$ of a chip, the involved crosscorrelation function now carries values between the discrete cross-correlation function in the "pure" chip-synchronous case and the continuous cross-correlation function in the "pure" chip-asynchronous case ${ }^{(2,4)}$. The new cross-correlation function is still discrete but now with fractional values taking from the set of $\{0,1 / s, 2 / s, \ldots, 3-2 / s\}$, according to (1). Taking these fractional values into consideration, the new hit probabilities can here be formulated as

$$
q_{k^{\prime}, l^{\prime}}=\frac{1}{2} \times \frac{h_{k^{\prime}, l^{\prime}}}{\phi \times(\phi-1) \times s^{2 w} \times N}
$$

which are indexed by $k^{\prime}$ and $l^{\prime} \in\{0,1 / s, 2 / s, \ldots, 3-2 / s\}$. The factor $1 / 2$ is due to OOK, $\phi-1$ represents the possible number of interfering codewords, out of a total of $\phi$ codewords in the code set, $N$ represents the number of possible time shifts in a codeword of length $N$, and $s^{2 w}$ represents all the possible pulse matching situations between two correlating codewords as there exist a total of $s^{w}$ pulse-shift positions in a codeword of weigh $w$. The term $h_{k^{\prime}, l}$, denotes the number of times of getting a $k^{\prime}$-hit in the preceding sub-chip and a $l^{\prime}$-hit in the current sub-chip, where $k^{\prime}$ and $l^{\prime} \in\{0,1 / s, 2 / s, \ldots, 3-2 / s\}$, which depends on the optical codes in use and can be computed numerically.

For example, if the $\left(w \times p_{1} p_{2} \cdots p_{m}, w, 1\right)$ CHPCs with $s=$ 2 are used, $\phi=p_{1} p_{2} \cdots p_{m}$ for a given integer $m \geq 1$ and $p_{m} \geq$ $p_{m-1} \geq \cdots \geq p_{2} \geq p_{1} \geq w$. The term $h_{k^{\prime}, l}$, for $k^{\prime}$ and $l^{\prime} \in\{0,1 / 2,1$, $3 / 2,2\}$, can be computed by i) first randomly pick two correlating codewords from the code set; ii) then build the $s^{w}$ possible sub-chip shifts in the $w$ pulses of these two codewords; iii) additionally build the $N$ possible cyclic chip shifts in one of the codeword; iv) for every combination of the $s^{w}$ sub-chip and $N$ chip shifts, count the number of times of getting a $k^{\prime}$-hit in the preceding sub-chip and a $l^{\prime}$-hit in the present sub-chip in the cross-correlation function, and then add to the associated $h_{k^{\prime}, l}$, term; v) finally repeat the above steps with all possible combinations of two correlating codewords in the code set.

In general, the chip-synchronous error probability $P_{\mathrm{e}, \mathrm{syn}, \mathrm{s}>1}$ caused by time skew with $s>1$ can be derived as ${ }^{(14)}$

$$
\begin{aligned}
P_{\mathrm{e}, \mathrm{syn}, s>1}= & \frac{1}{2} \sum_{i=0}^{2 w}(-1)^{2 w-i}\left(\begin{array}{c}
2 w \\
i
\end{array}\right) \\
& \times\left[\sum_{k=0}^{\lceil 3-2 / s\rceil\lceil 3-2 / s\rceil} \sum_{l=0}^{\left(\begin{array}{c}
i \\
k+l
\end{array}\right)} \frac{\left(\begin{array}{c}
2 w \\
k+l
\end{array}\right)}{k} q_{k, l}\right]^{K-1}
\end{aligned}
$$


where $K$ denote the number of simultaneous users. The rational of the derivation of (3) follows that of the pure chip-asynchronous analysis ${ }^{(2,}{ }^{4)}$. Under the pure chipasynchronous assumption, the cross-correlation value becomes a continuous function of time because the value is the amount of (partial) pulse overlap due to the relative time-shift between the two correlating codewords ${ }^{(4, \text { p. 36) }}$. As a result, the "asynchronous" cross-correlation function involves partial overlap of pulses found in two consecutive chips. Thus, $q_{k, l}$ is defined as the probability of the crosscorrelation value in the preceding chip equal to $k \in\left[0, \lambda_{\mathrm{c}}=1\right]$ and the crosscorrelation value in the present chip equal to $l$ $\in\left[0, \lambda_{\mathrm{c}}=1\right]$, as if it is under the pure chip-synchronous assumption. For $\lambda_{\mathrm{c}}=1$ optical codes, these hit probabilities arerelated by $q_{1,0}=q_{0,1}, q_{1}=w^{2} /(2 L N), q_{1,1}=w(w-1) /[2 N(N-1)]$, $q_{1}=0.5 \Sigma_{i=0}^{1} \Sigma_{j=0}^{1}(i+j) q_{i, j}$, and $\Sigma_{i=0} \Sigma_{j=0}^{1} q_{i, j}^{1}=1^{(2,4)}$.

To apply the new hit probabilities in (2) to (3), a mapping of $q_{k^{\prime}, l^{\prime}}$ to $q_{k, l}$ is needed and can be derived as

$$
\begin{aligned}
& q_{k, l}=\sum_{\operatorname{all}\left(k^{\prime}=k, l^{\prime}=l\right)} q_{k^{\prime}, l^{\prime}} \\
& +\sum_{\substack{\left.\operatorname{all}\left\{\left[k^{\prime},\right], k^{\prime}\right]\right\}=k \\
k^{\prime} \neq k}} \sum_{\operatorname{all}\left(l^{\prime}=l\right)}\left(1-\left|k-k^{\prime}\right|\right) q_{k^{\prime}, l} \\
& +\sum_{\operatorname{all}\left(k^{\prime}=k\right)} \sum_{\substack{\left.\operatorname{all}\left\{\left\{l_{i}^{\prime}\right], l_{i} l^{\prime}\right]\right\}=l \\
l \neq l}}\left(1-\left|l-l^{\prime}\right|\right) q_{k^{\prime}, l^{\prime}} \\
& +\sum_{\operatorname{all}\left\{\left[k^{k}\right],\left[k^{\prime}\right]\right\}=k} \sum_{\substack{k \neq k \\
k \neq k}} \\
& \frac{\left[\left(1-\left|k-k^{\prime}\right|\right)+\left(1-\left|l-l^{\prime}\right|\right) q_{k^{\prime}, l^{\prime}}\right.}{4}
\end{aligned}
$$

for $k^{\prime} \in\{0,1 / s, 2 / s, \ldots, 3-2 / s\}, l^{\prime} \in\{0,1 / s, 2 / s, \ldots, 3-2 / s\}, k$ $\in\{0,1,2, \ldots,\lceil 3-2 / s\rceil\}, l \in\{0,1,2, \ldots,\lceil 3-2 / s\rceil\},(k, l) \neq(0$, 0 ), and $\left(k^{\prime}, l^{\prime}\right) \neq(0,0)$, where $\lceil\cdot\rceil$ is the ceiling function and $\left.L \cdot\right\rfloor$ is the floor function.

By setting $s=1$ in (3), the hard-limiting error probability is then degenerated to

$$
P_{\text {e,asyn }}=\frac{1}{2} \sum_{i=0}^{2 w}(-1)^{2 w-i}\left(\begin{array}{c}
2 w \\
i
\end{array}\right)\left[\sum_{k=0}^{1} \sum_{l=0}^{1} \frac{\left(\begin{array}{c}
i \\
k+l
\end{array}\right)}{\left(\begin{array}{c}
2 w \\
k+l
\end{array}\right)} q_{k, l}\right]^{K-1}
$$

which is identical to the hard-limiting error probability of "chip-asynchronous" O-CDMA systems derived in ${ }^{(10)}$, thus verifying the validity of (3). In other words, the "chipasynchronous," hard-limiting error probability $P_{\text {e,asyn }}$ of 1-D

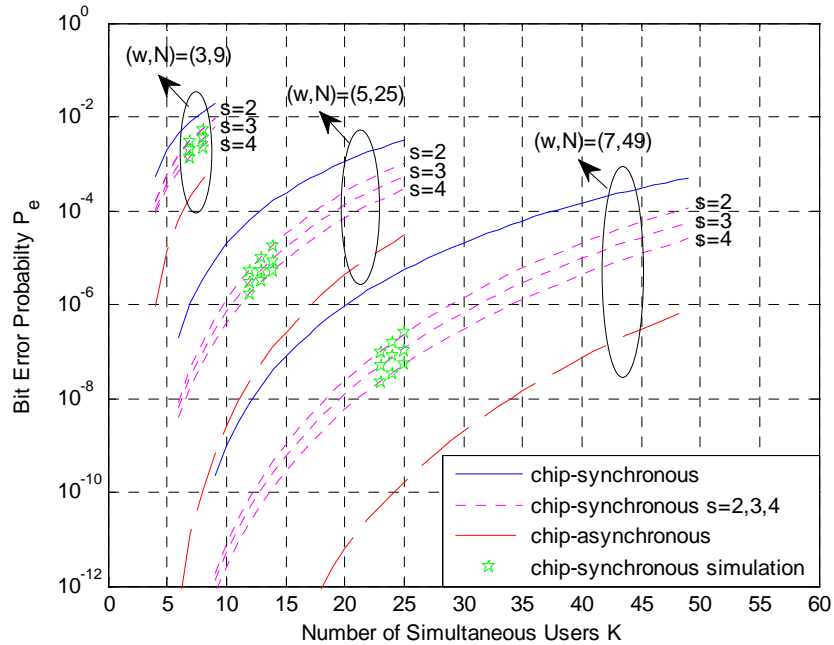

Fig. 3 Hard-limiting error probabilities, $P_{\mathrm{e}, \mathrm{syn}, \mathrm{s}>1}$ of (3), $P_{\text {e,asyn }}$ of (5), and $P_{\text {e,syn }}$ of (6), of the $(L \times N, w, 1)$ CHPCs versus the number of simultaneous users $K$, where $w=L=\{3$, $5,7\}, N=\{9,25,49\}$, and $s=\{2,3,4\}$.

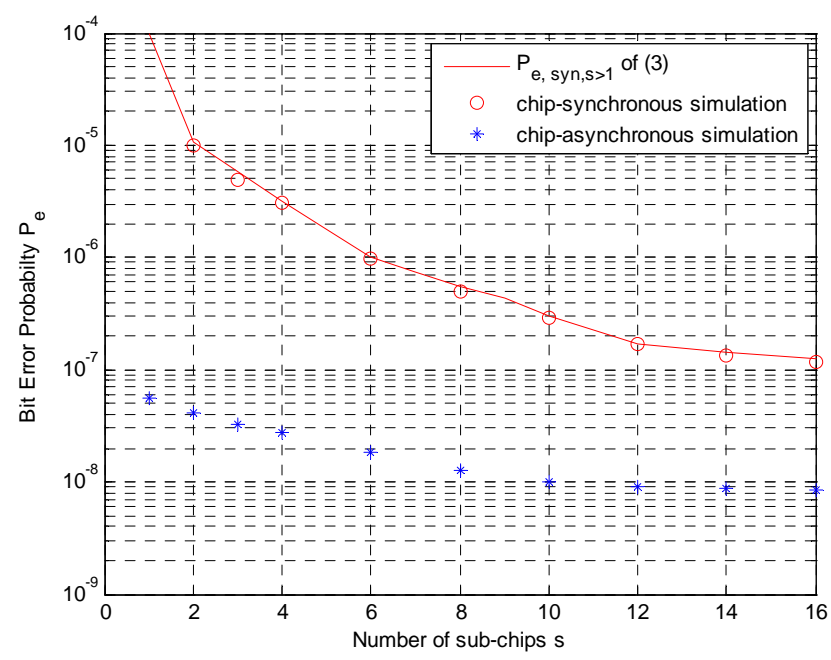

Fig. 4 Hard-limiting error probability, $P_{\mathrm{e}, \mathrm{syn}, \mathrm{s}>1}$ of (3), of the $(5 \times 25,5,1)$ CHPCs versus $s$ with $K=13$.

and 2-D optical codes with $\lambda_{\mathrm{c}}=1$ corresponds to the special case of $s=1$ in (3).

For reference, the "chip-synchronous" hard-limiting error probability of 1-D and 2-D optical codes with $\lambda_{\mathrm{c}}=1$ was given by ${ }^{(2,4)}$

$$
P_{\mathrm{e}, \mathrm{syn}}=\frac{1}{2} \sum_{i=0}^{w}(-1)^{i}\left(\begin{array}{c}
w \\
i
\end{array}\right)\left[1-\frac{q_{1}}{w}\right]^{K-1}
$$

where $q_{1}=w^{2} /(2 L N)$ denotes the probability of getting 1-hits in the cross-correlation function.

\section{Numerical Results}

In Figure 3, the hard-limiting error probabilities, $P_{\mathrm{e}, \mathrm{syn}, \mathrm{s}>1}$ of (3), $P_{\mathrm{e}, \text { asyn }}$ of (5), and $P_{\mathrm{e}, \mathrm{syn}}$ of (6), of the ( $L \times N, w$, 
1) CHPCs are plotted against the number of simultaneous users $K$, where $w=L=\{3,5,7\}, N=\{9,25,49\}$, and $s=\{2,3$, 4 \}. In general, the performance (i.e., $P_{\mathrm{e}}$ ) gets worse as $K$ increases due to stronger MAI. The code performance improves with $L, N, w$, and $s$ because the increment of $L$ or $N$ reduces the hit probabilities, the increment of $w$ increases the autocorrelation peak, and the hit probabilities reduced with increasing $s$. For a given set of $(w, N)$ values, the dotted curves of $P_{\mathrm{e}, \mathrm{syn}, \mathrm{s}>1}$ are bounded by the solid curve of $P_{\mathrm{e}, \text { syn }}$ and the dashed curve of $P_{\mathrm{e}, \text { asyn }}$. This is because these two curves correspond to the extreme cases of pure chipsynchronism and chip-asynchronism, respectively. Also shown in Figure 3 are the computer-simulation results (i.e., asterisks) of the hard-limiting error probabilities with the same code parameters as the corresponding theoretical (solid, dotted, and dashed) curves. Both theoretical and computer-simulation results (i.e., dotted curves vs. asterisks) match closely at various $s$ and $(w, N)$ values, thus validating the accuracy of the analytical model derive in (3).

Figure 4 plots the hard-limiting error probability, $P_{\mathrm{e}, \mathrm{syn}, \mathrm{s}>1}$ of $(3)$, of the $(5 \times 25,5,1)$ CHPCs as a function of $\mathrm{s}$ with $K=13$. In general, the red curve achieves better performance (i.e., lower $P_{\mathrm{e}}$ ) as $s$ increases because more sub-chips increases the number of possible locations for the pulses in each codeword, thus reducing the hit probabilities. Also shown in the figure are the computer-simulation results under the chip-synchronous (represented as circles) and chip-asynchronous (represented as asterisks) assumptions. The circles match closely with the red curve of $P_{\mathrm{e}, \mathrm{syn}, \mathrm{s}>1}$ from (3). The asterisks represents the results when chipasynchronism is applied in the simulation, in which a user begins codeword transmission at any time. In summary, by using different number of sub-chips (i.e., $s \in[2,16]$ ), the error probability is improved by 2-3 orders of magnitude as shown in the red curve. From the chip- asynchronous simulation results (i.e., asterisks), the error probability of $s=$ 16 is about 6 times better than that of $s=1$.

\section{Conclusions}

In this paper, a new analytical model for the time skew of optical pulses in O-CDMA codewords due to environmental changes was investigated. The hard-limiting performance of such a O-CDMA system was formulated, illustrated with a numerical example, and validated by computer simulation. Our study showed that larger number of sub-chips, $s$, increased the number of possible locations for the pulses in a codeword, thus reducing the hit probabilities and amount of MAI contributed by other simultaneous users. As a result, the performance improved as $s$ got larger. This new finding is unconventional in the sense that an O-CDMA system can make use of the time skew to improve performance.

\section{Acknowledgment}

This work was supported in part by the Ministry of Science and Technology of Republic of China under Grants NSC 102-2221-E-005-006-MY3, MOST 103-2221-E-239009 and MOST 103-2221-E-005-028, in part by the U.S. Defense Advance Research Projects Agency under Grant MDA972-03-1-0006, and in part by the Faulty Development and Research Grants of Hofstra University.

\section{References}

(1) Wing C. Kwong, Philippe A. Perrier, and Paul R. Prucnal: "Performance Comparison of Asynchronous and Synchronous Code-Division Multiple-Access Techniques for Fiber-Optic Local Area Networks", IEEE Trans. Commun., Vol. 39, No. 11, pp. 16251634, 1991

(2) Guu-Chang Yang and Wing C. Kwong: Prime Codes with Applications to CDMA Optical and Wireless Networks, Norwood, MA: Artech House, 2002

(3) Paul R. Prucnal: Optical Code Division Multiple Access: Fundamental and Applications, ed., Boca Raton, FL: Taylor \& Francis, 2006

(4) Wing C. Kwong and Guu-Chang Yang: Optical Coding Theory with Prime, New York: CRC Press, 2013

(5) Uri N. Griner and Shlomi Arnon: "Multiuser Diffuse Indoor Wireless Infrared Communication Using Equalized Synchronous CDMA", IEEE Trans. Commun., Vol. 54, No. 9, pp. 1654-1662, 2006

(6) Babak M. Ghaffari, Mehdi D. Matinfar, and Jawad A. Salehi: "Wireless Optical CDMA LAN: Digital Implementation Analysis", IEEE J. Select. Areas. Commun., Vol. 27, No. 9, pp. 1676-1686, 2009

(7) Chung-Yu Chang, Guu-Chang Yang, Cheng-Yuan Chang, and Wing C. Kwong: "Study of A Diversity OCDMA Scheme for Optical Wireless," J. Lightw. Technol., Vol. 30, No. 10, pp. 1549-1558, 2012

(8) Je-Wei Su, Guu-Chang Yang, Cheng-Yuan Chang, and Wing C. Kwong: "Design and Analysis of Generalized Diversity Optical CDMA Systems", J. Lightw. Technol., Vol. 31, No. 8, pp. 1217-1226, 2013 
(9) Chia-Cheng Hsu, Yi-Chun Chang, Guu-Chang Yang, Chun-Liang Chang and Wing C. Kwong: "Performance Analysis of 2-D O-CDMA Codes Without the Chip-Synchronous Assumption", IEEE J. Sel. Areas Commun., Vol. 25, No. 6, pp. 135-143, 2007

(10)Chia-Cheng Hsu, Guu-Chang Yang, and Wing C. Kwong: "Hard-Limiting Performance Analysis of 2-D Optical Codes Under the Chip-Asynchronous Assumption", IEEE Trans. Commun., Vol. 56, No. 5, pp. 762768, 2008

(11) Tolulope B. Osadola, Siti K. Idris, Ivan Glesk, and Wing C Kwong: "Effect of Variations in Environmental Temperature on 2D-WH/TS OCDMA Code Performance", J. Opt. Commun. Netw., Vol. 5, No. 1, pp. 68-73, 2013

(12) Chieh-Hung Hsieh, Guu-Chang Yang, Cheng-Yuan Chang, and Wing C. Kwong: "Multilevel Prime Codes for Optical CDMA Systems", J. Opt. Commun. Netw., Vol. 1, No. 7, pp. 600-607, 2009

(13) Cho-Cheng Sun, Guu-Chang Yang, Chen-Pin Tu, Cheng-Yuan Chang, and Wing C. Kwong: "Extended Multilevel Prime Codes for Optical CDMA", IEEE Trans. Commun., vol. 58, no. 5, pp. 1344-1350, 2010

(14) Yen-Ting Lin, Guu-Chang Yang, Cheng-Yuan Chang, and Wing C. Kwong: "Design and Analysis of OpticalCDMA Systems Using A New Code-Shifting Technique", submitted to IEEE Trans. Commun. 